BULLETIN OF THE

AMERICAN MATHEMATICAL SOCIETY

Volume 80, Number 1, January 1974

\title{
BRANCHED AND FOLDED PARAMETRIZATIONS OF THE SPHERE
}

BY GEORGE K. FRANCIS

Communicated by Glen Bredon, May 15, 1973

0 . This study is addressed to the following genre of topological problems. Let $\Pi$ be a subset of a manifold $W$ and $\varphi: \Sigma \rightarrow \Pi$ be a parametrization of $\Pi$ by a manifold collection $\Sigma$. We seek a factorization $\Sigma \rightarrow^{i} M \rightarrow^{F} W$, $\varphi=F \circ i$, where $i$ is an inclusion of $\Sigma$ in a manifold $M$ of the same dimension as $W$ and $F$ is a map in a certain class, such that the invariants of $(W, \Pi, \varphi)$ in some reasonable sense determine $(M, F, i)$ up to topological equivalence. For instance, let $\Pi$ be a closed, but not necessarily simply closed polygon in the complex plane $W, \Sigma$ the extended real line and $F$ a Schwarz-Christoffel transformation of the Gaussian upper half plane $M$, such that the image $[\varphi]$ of $\varphi=F \mid \Sigma$ coincides with $\Pi$. Necessary and sufficient conditions for $\Pi$ to bound a conformal, or more generally, a holomorphic image of a disc were first given by Titus [11]. In view of the Stoilow-Whyburn [16] theory, it proved more convenient to use light open maps $F$ such that $\varphi$ is a regular parametrization of a smooth, closed curve $\Pi$. If the curve lies in general position, the conditions can be expressed in terms of the Whitney [14]-Titus [10] intersection sequence, which is a combinatorial structure on the set of signed self-intersection points $X(\varphi)$ of $\Pi$. In the last decade considerable progress has been made in the direction of relaxing the specialized aspects of the Picard-Loewner problem solved by Titus. We present here some current work, the precise formulation of some technical definitions and proofs have or will appear elsewhere.

1. Let $M$ denote a smooth, compact oriented surface, possibly with boundary $\partial M$, and $W$ a smooth, oriented surface without boundary, but with base point $\infty$. We admit smooth maps $F: M \rightarrow W$ which in the vicinity of a point $m \in M$ is locally smoothly equivalent to one of the following canonical plane maps near the origin:

$m \in B$ is a branch point $\left(w=(x+i y)^{v}, v>1\right)$ of valence $v$,

$m \in C$ is a fold point $\left(w=x^{2}+i y\right)$,

$m \in K$ is a cusp point $\left(w=x^{3}-x y+i y\right)$,

$m \in P=F^{-1}(\infty)$ is a simple pole point $\left(w=(x+i y)^{-1}\right)$,

$m \in J=\partial M$ is a border point $(w=x+i y, y \geqq 0)$,

$m \in M_{0}$ is a regular point $(w=x+i y)$.

AMS (MOS) subject classifications (1970). Primary 30A48, 57D45; Secondary 53A05, 55A10, 58C25. 
Note that $K \subset \mathrm{Clos} C$ in such a way that $Z=K \cup C$ is a collection of embedded circles, $J$ a collection of boundary circles, and $B \cup P$ a discrete set of points in $M$. Thus $\Sigma=B \cup P \cup J \cup Z$ defines a stratification of $M$. Each component $M^{(j)}$ of $M-(J \cup Z)$ has a sign $e_{j}= \pm 1$ according to whether $F \mid M^{(\jmath)}$ is orientation preserving or reversing. Since $e$ changes sign across a circle of $Z$, we may orient $J \cup Z$ so that $e_{j} M^{(j)}$ lies to the left of $\partial M^{(j)}$.

2. The problem of reconstructing $F$ from $\varphi=F \mid \Sigma$ is greatly simplified if $W$ is taken to be the oriented sphere $S^{2}$. Let $E=S^{2}-\{\infty\}$ denote the oriented plane. Let $\tau$ denote a tangent winding measure on oriented regular curves, normalized to equal unity on the positive circle. $\tau$ can be extended to piecewise regular curves provided their singularities are corners. Note that the valence $\nu$, the measure $\tau$ and the cardinality \# are additive. The Euler characteristic of $M$ is constrained by $F$ to be

$$
\chi(M)=2 \# P+\# B-v(F \mid B)+2 \tau(F \mid C)+\tau(F \mid J) .
$$

This is an elementary synthesis of well-known formulas. For $J=Z=\varnothing$ it is the Hurwitz [6]-Riemann formula

$$
\begin{aligned}
\operatorname{deg}(F) \chi\left(S^{2}\right)-\chi(M) & =v(F \mid B)-\# B \\
& =\operatorname{deg}(F) \# F(B)-\# F^{-1} F(B) .
\end{aligned}
$$

For $M$ of genus zero and $Z$ empty, it is equivalent to a formula of Morse [9]-Heins, because $F$ is light-open and satisfies their first boundary condition. For $B=J=\varnothing, F$ is an "excellent" map of Whitney [15] and (1) is a specialization of Levine's [7] formula. In particular, there are an even number of cusps (Thom [13], Haefliger [5]). Indeed, on a component circle $S$ of $Z, 2 \tau(F \mid S)=\hat{\tau}(F \mid S)$ is the projective tangent winding number which is $\bmod (2)$ congruent to $\# K(F \mid S)$. Setting $\beta=\# P, \gamma=\operatorname{genus}(M)$, $\rho=\# J, \tau=\tau(F \mid J), \hat{\tau}=\hat{\tau}(F \mid Z)$ and $\mu=v(F \mid B)-\# B$, (1) becomes

$$
2+\mu=2 \beta+2 \gamma+\rho+\tau+\hat{\tau}, \quad \mu, \beta, \gamma, \rho \geqq 0 .
$$

3. Let $N_{r}$ be a normal tubular neighborhood of $\Sigma$, with ${ }^{\circ} N_{0}=\Sigma$, $M_{r}=M-N_{r}$ and $\Sigma_{r}=\partial M_{r}$. For some sufficiently small positive function $r(\theta), \theta \in \Sigma, F \mid M_{r}$ is an immersion into the plane, for which $f=F \mid \Sigma_{r}$ lies in general position. Choose a maximal tree $\psi$ in the dual graph of $[f]$, oriented towards the infinite complementary component. Then $F$ induces a combinatorial structure $\mathscr{A}$, called an assemblage, on $X(f, \psi)=[f] \cap[\psi]$.

The central problem is to decide what an assemblage should be in order that it classifies the map $F$. Blank [2], Marx [8], Francis [4], Bailey [1] and Troyer [12] have constructed assemblages for plane immersed discs $(P=B=Z=\varnothing)$, plane properly interior discs $(P=Z=\varnothing)$, spherically immersed discs $(B=Z=\varnothing)$, plane immersions $(P=B=Z=\varnothing)$ of the 
discs with handles, respectively with holes. These methods extend to arbitrary plane immersions $(B=Z=\varnothing)$, and thus yield assemblages for $F$ restricted to the components of $M_{r}$. Since $N_{r}$ is the disjoint union of annuli and discs, a signed graph dictates how the subassemblages fit together to form $\mathscr{A}$.

4. Conversely, given a finite collection $[f]$ of plane immersed circles in general position, the tree $\psi$ and an assemblage $\mathscr{A}$ on $X(f, \psi)$, then there exists an admissible map $F: M \rightarrow S^{2}$ and a tube $N_{r}$ of its stratification $\Sigma$ such that $F \mid \Sigma_{r}=f$. Moreover, $\mathscr{A}$ is the assemblage induced by $F, N_{r}$ and $\psi$, and $\varphi=F \mid \Sigma$ lies in general position. That is, $[\varphi]$ is locally equivalent to the locus described by $x^{2}+y^{2}=0$ (isolated point), $x y=0$ (node), $x^{3}=y^{2}$ (cusp) or $x=0$ (regular arc).

The problem of constructing $F$ on the components of $N_{r}$ is really a homotopy problem. A prototype [3] is the folded ribbon theorem: Two regularly homotopic plane immersed circles bound the admissible image of an annulus with a single, concentric fold circle. The methods of that study also show, for instance, that two spherically immersed circles bound an admissible annulus with at most one fold, and one or no cusps, depending on whether the curves are not or are regularly homotopic. Thus every spherically immersed circle bounds an admissible disc with at most one fold circle if the curve is regularly homotopic to a circle on the sphere. If not, it is regularly homotopic to the figure eight and the extension has exactly one cusp. If the possible number of cusps is increased, similar theorems hold in the plane. For those components of $N_{r}$ assigned to contain a single branch point, Marx' assemblages are well suited.

5. If $F: M \rightarrow S^{2}$ is an admissible map with $\varphi=F \mid \Sigma$ in general position, then every assemblage $\mathscr{A}$ obtained from a suitable narrow tube $N_{r}$ and properly chosen tree $\psi$ induces an orientation preserving automorphism $H: M \rightarrow M, H \mid \Sigma=1_{\Sigma}$, such that $F_{\mathscr{A}}=F \circ H$.

One chooses $N_{r}$ so narrow that for $m \in P \cup B, F \mid e(m) N_{r}(m)$ is an orientation preserving embedding, respectively a canonical properly interior map with single branch point $m$. For a circle $S$ of $J \cup Z, F \mid N_{r}(S)$ is an immersed annulus with parallel borders, respectively an admissible map of the annulus with borders parallel to the singular curve $\varphi(S)$ except, of course, near the cusps, where one loops about the cusp image in a canonical way. These constructions permit a tree $\psi$ to be so carefully chosen that its $F$-lift decomposes $M$ into cells with disjoint interiors on which $F$ is univalent. This cell decomposition induces the equivalence $H$.

6. We summarize by giving a solution to the following Picard-Loewner problem. Let $\Pi$ be a finite spherical collection of points and smooth 
closed curves possessing continuously turning tangents. A $C^{1}$-parametrization $\varphi: \Sigma \rightarrow \Pi$ by a collection of points and circles is called a seed if it is regular on the curves except at their cusps. The seed grows $\Pi$ into a one parameter family $\Pi_{r}$ of immersed circles. Pick $[f]=\Pi_{r}$ to lie in general position. $\varphi$ has an admissible extension $\Sigma \rightarrow^{i} M \rightarrow^{F} S^{2}$ precisely if $X(f, \psi)$ has an assemblage $\mathscr{A}$. If $[\varphi]=\Pi$ is already in general position, the assemblages combinatorially classify all topologically distinct extensions.

7. We close this announcement with a simple example. Let $\Pi$ be the connected sum of two Milnor paisley curves in the plane $E$. Then $\Sigma$ is a circle and the only $M$ satisfying (1) is the disc $D$ with boundary $S$ or the
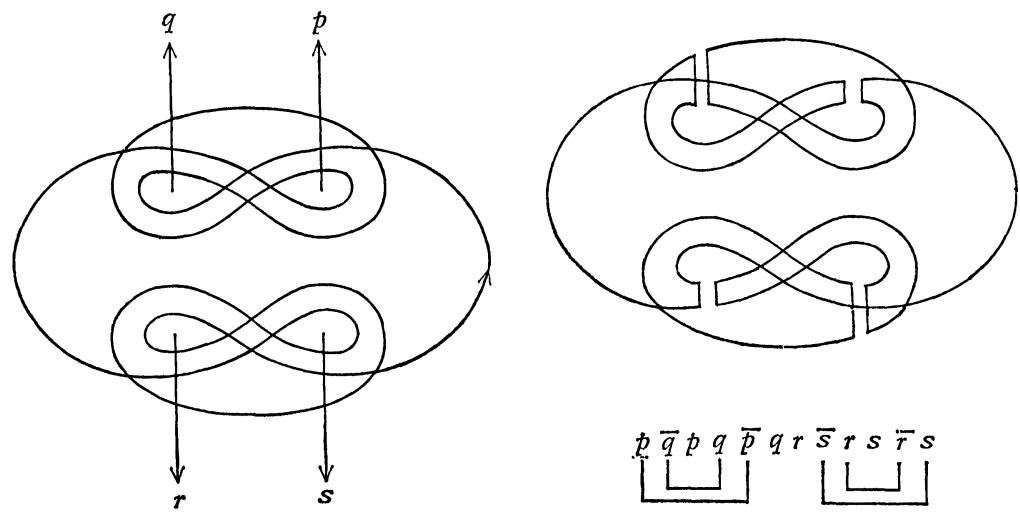

sphere with equator $S$. In the latter case both subassemblages are assemblages for the former case. We let $\varphi=f: \Sigma \rightarrow \Pi$ be a regular parametrization. Consider the dual tree $\psi$, four spokes to $\infty$ are indicated. How the lozenge shaped cells are attached to $\psi$ is immaterial. An assemblage can be represented as follows (compare with [2]). List the crossings $X(f, \psi)$ of $f$ with the four spokes in the circular order encountered: $p \bar{q} p q \bar{p} q r \bar{s} r s \bar{r} s$. The bar indicates a clockwise crossing. The assemblage pairs eaçh barred letter with one of the two unbarred letters of the same denomination, but so that no pair splits another. Thus there are exactly 4 possibilities. The decomposition into embedded discs induced by one of these is indicated in the second picture.

The bilateral symmetry of $\Pi$ shows how one might obtain the coarser classification of admissible maps under the equivalence $F \circ H=K \circ G$, where $(H, K)$ are $(\Sigma, \Pi)$-invariant diffeomorphisms of $D \times E$. If both $(H, K)$ leave one point on $\Sigma$ fixed and preserve orientation, there are still four. If both $(H, K)$ preserve orientation, there are three, and if $(H, K)$ are merely orientation coherent, there are but two equivalence classes. 


\section{REFERENCES}

1. K. D. Bailey, Extending closed plane curves to immersions of a disc with $n$ handles, Dissertation, University of Illinois, Chicago, Ill., 1973.

2. S. J. Blank, Extending immersions of the circle, Dissertation, Brandeis University, 1967. cf. V. Poenaru, Exposé 342, Séminaire Bourbaki 1967/68, Benjamin, New York, 1969.

3. G. K. Francis, The folded ribbon theorem, Dissertation, University of Michigan, Ann Arbor, Mich , 1967; cf. Trans. Amer. Math. Soc. 141 (1969), 271-303. MR 39 \#4863.

4. - Spherical curves that bound immersed discs, Proc. Amer. Math. Soc. 41 (1973), 87-93.

5. A. Haefliger, Quelques remarques sur les applications différentiables d'une surface dans le plan, Ann. Inst. Fourier (Grenoble) 10 (1960), 47-60. MR 22 \#7145.

6. A. Hurwitz, Ueber Riemannsche Flaechen mit gegebenen Verzweigungspunkten, Math. Ann. 39 (1891), 1-61.

7. H. Levine, Elimination of cusps, Topology 3 (1965), suppl. 2, 263-296. MR 31 \#756.

8. M. L. Marx, Extensions of normal immersions of $S^{1}$ into $R^{2}$, Trans. Amer. Math. Soc. (to appear).

9. M. Morse, Topological methods in the theory of functions of a complex variable, Ann. of Math. Studies, no. 15, Princeton Univ. Press, Princeton, N.J., 1947. MR 9, 20.

10. C. J. Titus, $A$ theory of normal curves and some applications, Pacific J. Math. 10 (1960), 1083-1096. MR 22 \#5014.

11. - The combinatorial topology of analytic functions on the boundary of a disc, Acta Math. 106 (1961), 45-64. MR 29 \#3652.

12. S. F. Troyer, Extending a boundary immersion to the disk with $n$ holes, Dissertation, Northeastern University, Boston, Mass., 1973.

13. R. Thom, Les singularités des applications différentiables, Ann. Inst. Fourier (Grenoble) 6 (1955-1956), 43-87. MR 19, 310.

14. H. Whitney, On regular closed curves in the plane, Compositio Math. 4 (1937), 276-284.

15. — On singularities of mappings of Euclidean spaces. I. Mappings of the plane into the plane, Ann. of Math. (2) 62 (1955), 374-410. MR 17, 518.

16. G. Whyburn, Topological analysis, Princeton Math. Series, no. 23, Princeton Univ. Press, Princeton, N.J., 1958. MR 20 \#6081.

Department of Mathematics, University of Illinois, Urbana, Illinois 61801 